ISSN 1855-3966 (printed edn.), ISSN 1855-3974 (electronic edn.)

ARS MATHEMATICA CONTEMPORANEA 18 (2020) 179-186

https://doi.org/10.26493/1855-3974.1894.37c

(Also available at http://amc-journal.eu)

\title{
Arc-transitive graphs of valency twice a prime admit a semiregular automorphism*
}

\author{
Michael Giudici \\ Department of Mathematics and Statistics, The University of Western Australia, \\ 35 Stirling Highway, Crawley, WA 6009, Australia \\ Gabriel Verret $\ddagger$ \\ Department of Mathematics, The University of Auckland, \\ Private Bag 92019, Auckland 1142, New Zealand
}

Received 1 January 2019, accepted 16 February 2020, published online 15 October 2020

\begin{abstract}
We prove that every finite arc-transitive graph of valency twice a prime admits a nontrivial semiregular automorphism, that is, a non-identity automorphism whose cycles all have the same length. This is a special case of the Polycirculant Conjecture, which states that all finite vertex-transitive digraphs admit such automorphisms.
\end{abstract}

Keywords: Arc-transitive graphs, polycirculant conjecture, semiregular automorphism.

Math. Subj. Class. (2020): 20B25, 05E18

\section{Introduction}

All graphs in this paper are finite. In 1981, Marušič asked if every vertex-transitive digraph admits a nontrivial semiregular automorphism [13], that is, an automorphism whose cycles all have the same length. This question has attracted considerable interest and a generalisation of the affirmative answer is now referred to as the Polycirculant Conjecture [4]. See [1] for a recent survey on this problem.

One line of investigation of this question has been according to the valency of the graph or digraph. Every vertex-transitive graph of valency at most four admits such an automorphism [7, 14], and so does every vertex-transitive digraph of out-valency at most three [9].

\footnotetext{
* Authors are grateful to the anonymous referees for their helpful suggestions.

${ }^{\dagger}$ The research of the first author was supported by the ARC Discovery Project DP160102323.

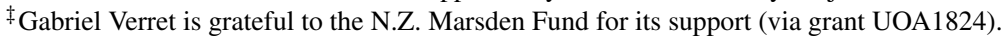

E-mail addresses: michael.giudici@uwa.edu.au (Michael Giudici), g.verret@auckland.ac.nz (Gabriel Verret)
} 
Every arc-transitive graph of prime valency has a nontrivial semiregular automorphism [10] and so does every arc-transitive graph of valency 8 [17]. Partial results were also obtained for arc-transitive graphs of valency a product of two primes [18]. We continue this theme by proving the following theorem.

Theorem 1.1. Arc-transitive graphs of valency twice a prime admit a nontrivial semiregular automorphism.

\section{Preliminaries}

If $G$ is a group of automorphisms of a graph $\Gamma$ and $v$ is a vertex of $\Gamma$, we denote by $G_{v}$ the stabiliser in $G$ of $v$, by $\Gamma(v)$ the neighbourhood of $v$, and by $G_{v}^{\Gamma(v)}$ the permutation group induced by $G_{v}$ on $\Gamma(v)$. We will need the following well-known results.

Lemma 2.1. Let $\Gamma$ be a connected graph and $G \leqslant \operatorname{Aut}(\Gamma)$. If a prime $p$ divides $\left|G_{v}\right|$ for some $v \in \mathrm{V}(\Gamma)$, then there exists $u \in \mathrm{V}(\Gamma)$ such that $p$ divides $\left|G_{u}^{\Gamma(u)}\right|$.

Proof. Since $p$ divides $\left|G_{v}\right|$, there exists an element $g$ of order $p$ in $G_{v}$. As $g \neq 1$, there are vertices not fixed by $g$. Among these vertices, let $w$ be one at minimal distance from $v$. Let $P$ be a path of minimal length from $v$ to $w$ and let $u$ be the vertex preceding $w$ on $P$. By the definition of $w$, we have that $u$ is fixed by $g$, so $g \in G_{u}$. On the other hand, $g$ does not fix the neighbour $w$ of $u$, so $g^{\Gamma(u)} \neq 1$ hence $\left|g^{\Gamma(u)}\right|=p$ and the result follows.

Lemma 2.2. Let $G$ be a permutation group and let $K$ be a normal subgroup of $G$ such that $G / K$ acts faithfully on the set of $K$-orbits. If $G / K$ has a semiregular element $K g$ of order $r$ coprime to $|K|$, then $G$ has a semiregular element of order $r$.

Proof. See for example [17, Lemma 2.3].

Lemma 2.3. A transitive group of degree a power of a prime p contains a semiregular element of order $p$.

Proof. In a transitive group of degree a power of a prime $p$, every Sylow $p$-subgroup is transitive. A non-trivial element of the center of this subgroup must be semiregular.

Recall that a permutation group is quasiprimitive if every non-trivial normal subgroup is transitive, and biquasiprimitive if it is transitive but not quasiprimitive and every nontrivial normal subgroup has at most two orbits.

\section{Arc-transitive graphs of prime valency}

In the most difficult part of our proof, the arc-transitive graph of valency twice a prime will have a normal quotient with prime valency. We will thus need a lot of information about arc-transitive graphs of prime valency, which we collect in this section. We start with the following result, which is [3, Theorem 5]:

Theorem 3.1. Let $\Gamma$ be a connected $G$-arc-transitive graph of prime valency $p$ such that the action of $G$ on $\mathrm{V}(\Gamma)$ is either quasiprimitive or biquasiprimitive. Then one of the following holds:

(1) G contains a semiregular element of odd prime order; 
(2) $|\mathrm{V}(\Gamma)|$ is a power of 2;

(3) $\Gamma=\mathrm{K}_{12}, G=\mathrm{M}_{11}$ and $p=11$;

(4) $|\mathrm{V}(\Gamma)|=\left(p^{2}-1\right) / 2 s$ and $G=\mathrm{PSL}_{2}(p)$ or $\mathrm{PGL}_{2}(p)$, where $p$ is a Mersenne prime and $s$ is a proper divisor of $(p-1) / 2$ but also a multiple of the product of the distinct prime divisors of $(p-1) / 2$;

(5) $|\mathrm{V}(\Gamma)|=\left(p^{2}-1\right) / s$ and $G=\mathrm{PGL}_{2}(p)$, where $p$ and $s$ are as in part (4), and $\Gamma$ is the canonical double cover of the graph given in (4).

(Recall that the canonical double cover of a graph $\Gamma$ is $\Gamma \times \mathrm{K}_{2}$.) We note that in cases (4) and (5), we must have $p \geqslant 127$, since this is the smallest Mersenne prime $p$ such that $(p-1) / 2$ is not squarefree. This fact will be used at the end of Section 4.

Corollary 3.2. Let $\Gamma$ be a connected $G$-arc-transitive graph of prime valency. Then one of the following holds:

(1) G contains a semiregular element of odd prime order;

(2) $|\mathrm{V}(\Gamma)|$ is a power of 2;

(3) G contains a normal 2-subgroup $P$ such that $(\Gamma / P, G / P)$ is one of the graph-group pairs in (3-5) of Theorem 3.1.

Proof. Suppose that $|\mathrm{V}(\Gamma)|$ is not a power of 2 . If $G$ is quasiprimitive or biquasiprimitive on $\mathrm{V}(\Gamma)$, then the result follows immediately from Theorem 3.1 (with $P=1$ in case (3)). We thus assume that this is not the case and let $P$ be a normal subgroup of $G$ that is maximal subject to having at least three orbits on $\mathrm{V}(\Gamma)$. In particular, $P$ is the kernel of the action of $G$ on the set of $P$-orbits. Hence $G / P$ acts faithfully, and quasiprimitively or biquasiprimitively on $\mathrm{V}(\Gamma / P)$. Since $\Gamma$ has prime valency, is connected and $G$-arctransitive, [12, Theorem 9] implies that $P$ is semiregular. We may thus assume that $P$ is a 2-group. (Otherwise $P$ contains a semiregular element of odd prime order.) If $G / P$ contains a semiregular element of odd prime order, then Lemma 2.2 implies that so does $G$. We may assume that this is not the case. Similarly, we may assume that $|\mathrm{V}(\Gamma / P)|$ is not a power of 2. (Otherwise, $|\mathrm{V}(\Gamma)|$ is a power of 2.) It follows that $\Gamma / P$ and $G / P$ are as is $(3-5)$ of Theorem 3.1 .

We will now prove some more results about the graphs that appear in (3-5) of Theorem 3.1. Let us first recall the notion of coset graphs. Let $G$ be a group with a subgroup $H$ and let $g \in G$ such that $g^{2} \in H$ but $g \notin \mathrm{N}_{G}(H)$. The graph $\operatorname{Cos}(G, H, H g H)$ has vertices the right cosets of $H$ in $G$, with two cosets $H x$ and $H y$ adjacent if and only if $x y^{-1} \in H g H$. Observe that the action of $G$ on the set of vertices by right multiplication induces an arc-transitive group of automorphisms such that $H$ is the stabiliser of a vertex. Moreover, every arc-transitive graph can be constructed in this way [16, Theorem 2].

Lemma 3.3. The graphs in (3) and (4) of Theorem 3.1 have a 3-cycle.

Proof. Clearly $\mathrm{K}_{12}$ has a 3-cycle so suppose that $\Gamma$ is one of the graphs given in (4). Let $G$ be as in Theorem 3.1 and let $v \in \mathrm{V}(\Gamma)$. Then $G$ is one of $\operatorname{PSL}_{2}(p)$ or $\operatorname{PGL}_{2}(p)$ and acts arc-transitively on $\Gamma$. In both cases, let $X=\mathrm{PSL}_{2}(p)$, so $G=X$ or $|G: X|=2$. By [3, Lemma 5.3], we have that $G_{v} \cong \mathrm{C}_{p} \rtimes \mathrm{C}_{s}$ if $G=\operatorname{PSL}_{2}(p)$, and $\mathrm{C}_{p} \rtimes \mathrm{C}_{2 s}$ if 
$G=\mathrm{PGL}_{2}(p)$. By [5, pp. 285-286], $\mathrm{PGL}_{2}(p)$ has a unique conjugacy class of subgroups of order $p$ and the normaliser of such a subgroup is isomorphic to $\mathrm{C}_{p} \rtimes \mathrm{C}_{p-1}$, which is the stabiliser in $\mathrm{PGL}_{2}(p)$ of a 1-dimensional subspace of the natural 2-dimensional vector space. The intersection of this subgroup with $X$ is isomorphic to $\mathrm{C}_{p} \rtimes \mathrm{C}_{(p-1) / 2}$, which has odd order. It follows that if $|G: X|=2$, then $G_{v}$ is not contained in $X$. Thus, in both cases, $X$ is transitive on $\mathrm{V}(\Gamma)$. Since $X$ is normal in $G, X_{v} \neq 1$ and $\Gamma$ has prime valency, it follows that $X$ is arc-transitive on $\Gamma$ and so $\Gamma=\operatorname{Cos}(X, H, H g H)$ where $H \cong \mathrm{C}_{p} \rtimes \mathrm{C}_{s}$ and $g \in X \backslash \mathrm{N}_{X}(H)$ such that $H g H$ is a union of $p$ distinct right cosets of $H$.

Since $H$ has a characteristic subgroup $Y$ of order $p$, it follows that $\mathrm{N}_{X}(H)$ normalises $Y$ and so $\mathrm{N}_{X}(H) \leqslant \mathrm{N}_{X}(Y) \cong \mathrm{C}_{p} \rtimes \mathrm{C}_{(p-1) / 2}$. Since $\mathrm{N}_{X}(Y) / Y$ is cyclic it follows that $\mathrm{N}_{X}(H)=\mathrm{N}_{X}(Y)$. Also note that $\mathrm{N}_{X}(H)$ is the stabiliser in $X$ of a 1-dimensional subspace and so the action of $X$ on the set of right cosets of $\mathrm{N}_{X}(H)$ is 2-transitive and the stabiliser of any two 1-dimensional subspaces is isomorphic to $\mathrm{C}_{(p-1) / 2}$. Let $x \in X$. The stabiliser in $X$ of the coset $H x$ is $H^{x}$ and so the orbit of $H x$ under $H$ has length $\left|H: H \cap H^{x}\right|$. In particular, $H$ fixes the coset $H x$ if and only if $x \in \mathrm{N}_{X}(H)$, and so $H$ fixes $\left|\mathrm{N}_{X}(H): H\right|=(p(p-1) / 2) / p s=(p-1) / 2 s$ points of $\mathrm{V}(\Gamma)$. Moreover, since the stabiliser of two 1-dimensional subspaces is isomorphic to $\mathrm{C}_{(p-1) / 2}$ it follows that if $x \notin \mathrm{N}_{X}(H)$ then $H \cap H^{x} \cong \mathrm{C}_{(p-1) / 2}$ and so $\left|H: H \cap H^{x}\right|=p$. Thus the points of $\mathrm{V}(\Gamma)$ that are not fixed by $H$ are permuted by $H$ in orbits of size $p$ and so for any $g \notin \mathrm{N}_{X}(H)$ we have that $H g H$ is a union of $p$ distinct right cosets of $H$.

For each $x \in \mathrm{N}_{X}(H)$ define the bijection $\lambda_{x}$ of $\mathrm{V}(\Gamma)$ by $H y \mapsto x^{-1} H y=H x^{-1} y$. Since $X$ acts on $\mathrm{V}(\Gamma)$ by right multiplication, we see that $\lambda_{x}$ commutes with each element of $X$. Moreover, $\lambda_{x}$ is nontrivial if and only if $x \notin H$. Let $C=\left\{\lambda_{x} \mid x \in \mathrm{N}_{G}(H)\right\} \leqslant$ $\operatorname{Sym}(\mathrm{V}(\Gamma))$. Since $X$ acts transitively on $\mathrm{V}(\Gamma)$ and $C$ centralises $X$, it follows from [6, Theorem 4.2A] that $C$ acts semiregularly on $\mathrm{V}(\Gamma)$. Now $|C|=\left|\mathrm{N}_{X}(H): H\right|=(p-1) / 2 s$ and $X \times C \leqslant \operatorname{Sym}(\mathrm{V}(\Gamma))$. Since $C \unlhd X \times C$, the set of orbits of $C$ forms a system of imprimitivity for $X \times C$ and hence for $X$. Moreover, since $C$ is semiregular, comparing orders yields that $C$ has $|\mathrm{V}(\Gamma)| /|C|=p+1$ orbits. One of these orbits is the set of fixed points of $H$ and $H$ transitively permutes the remaining $p$ orbits of $C$. In particular, it follows that $C$ transitively permutes the nontrivial orbits of $H$ and so the isomorphism type of $\Gamma$ does not depend on the choice of the double coset $\mathrm{HgH}$.

Let $Z$ be the subgroup of scalar matrices in $\mathrm{SL}_{2}(p)$ and let $\hat{H}$ be the subgroup of the stabiliser in $\mathrm{SL}_{2}(p)$ of the 1-dimensional subspace $\langle(1,0)\rangle$ such that $H=\hat{H} / Z$. Note that $\hat{h}=\left(\begin{array}{ll}1 & 0 \\ 1 & 1\end{array}\right) \in \hat{H}$ and let $\hat{g}=\left(\begin{array}{cc}0 & 1 \\ -1 & 0\end{array}\right)$. In particular, letting $h=\hat{h} Z$ and $g=\hat{g} Z$ we have that $g \notin \mathrm{N}_{X}(H)$ and so we may assume that $\Gamma=\operatorname{Cos}(X, H, H g H)$. Now $H g$ and $H g h$ are both adjacent to $H$ and one can check that $g(g h)^{-1}=h g h \in H g H$ and so $\{H, H g, H g h\}$ is a 3 -cycle in $\Gamma$.

Definition 3.4. Let $\Gamma$ be a graph and let $S_{0}$ be a subset of $\mathrm{V}(\Gamma)$. Let $S=S_{0}$. If a vertex $u$ outside $S$ has at least two neighbours in $S$, add $u$ to $S$. Repeat this procedure until no more vertices outside $S$ have this property. If at the end of the procedure, we have $S=\mathrm{V}(\Gamma)$, then we say that $\Gamma$ is dense with respect to $S_{0}$.

It is an easy exercise to check that denseness is well-defined.

Corollary 3.5. Let $\Gamma$ be a graph in (3) or (4) of Theorem 3.1 and let $S_{0}=\{u, v\}$ be an edge of $\Gamma$. Then $\Gamma$ is dense with respect to $S_{0}$. 
Proof. Since $\Gamma$ is arc-transitive of prime valency $p$, the local graph at $v$ (that is, the subgraph induced on $\Gamma(v)$ ) is a vertex-transitive graph of order $p$ and thus vertex-primitive. By Lemma 3.3, $\Gamma$ has a 3-cycle so the local graph has at least one edge and thus must be connected. It follows that, running the process described in Definition 3.4 starting at $S=S_{0}$, eventually $S$ will contain all neighbours of $v$. Repeating this argument and using connectedness of $\Gamma$ yields the desired conclusion.

The following is immediate from Definition 3.4.

Lemma 3.6. Let $\Gamma$ be a graph and $S_{0}$ be a set of vertices such that $\Gamma$ is dense with respect to $S_{0}$. Then the canonical double cover of $\Gamma$, with vertex-set $\mathrm{V}(\Gamma) \times\{0,1\}$, is dense with respect to $S_{0} \times\{0,1\}$.

Proof. Let $S_{i}$ be the sequence of subsets of $\mathrm{V}(\Gamma)$ obtained when running the procedure from Definition 3.4 starting with $S_{0}$ and ending with $S_{n}$ for some $n$. Since $\Gamma$ is dense with respect to $S_{0}$, we have $S_{n}=\mathrm{V}(\Gamma)$. For $i \in\{1, \ldots, n\}$, let $v_{i}=S_{i} \backslash S_{i-1}$. (In other words, $v_{1}$ is the first vertex added to $S_{0}$ to get $S_{1}$, then $v_{2}$ is added to $S_{1}$ to get $S_{2}$, etc.)

Now, let $\Gamma^{\prime}=\Gamma \times \mathrm{K}_{2}$ be the canonical double cover of $\Gamma$ and let $S_{0}^{\prime}=S_{0} \times\{0,1\} \subseteq$ $\mathrm{V}\left(\Gamma^{\prime}\right)$. We now run the procedure from Definition 3.4 starting at $S_{0}^{\prime}$. At the first step, we note that, since $v_{1}$ was added to $S_{0}$, it must have at least two neighbours in $S_{0}$, say $u_{1}$ and $w_{1}$. It follows that both $\left(v_{1}, 0\right)$ and $\left(v_{1}, 1\right)$ also have at least two neighbours in $S_{0}^{\prime}$ (for example, $\left(u_{1}, 1\right)$ and $\left(w_{1}, 1\right)$, and $\left(u_{1}, 0\right)$ and $\left(w_{1}, 0\right)$, respectively). We thus add $\left(v_{1}, 0\right)$ and $\left(v_{1}, 1\right)$ to $S_{0}^{\prime}$ to get $S_{1}^{\prime}=S_{0}^{\prime} \cup\left\{\left(v_{1}, 0\right),\left(v_{1}, 1\right)\right\}$. Note that $S_{1}^{\prime}=S_{1} \times\{0,1\}$. We then repeat this procedure, preserving the condition $S_{i}^{\prime}=S_{i} \times\{0,1\}$ at each iteration. At the end of this process, we have $S_{n}^{\prime}=S_{n} \times\{0,1\}=\mathrm{V}(\Gamma) \times\{0,1\}=\mathrm{V}\left(\Gamma^{\prime}\right)$ and so $\Gamma^{\prime}$ is dense with respect to $S_{0} \times\{0,1\}$.

\section{Proof of Theorem 1.1}

Let $p$ be a prime, let $\Gamma$ be an arc-transitive graph of valency $2 p$ and let $G=\operatorname{Aut}(\Gamma)$. We may assume that $\Gamma$ is connected. If $G$ is quasiprimitive or bi-quasiprimitive, then $G$ contains a nontrivial semiregular element, by [8, Theorem 1.2] and [10, Theorem 1.4]. We may thus assume that $G$ has a minimal normal subgroup $N$ such that $N$ has at least three orbits. In particular, $\Gamma / N$ has valency at least 2 .

If $N$ is nonabelian, then $G$ has a nontrivial semiregular element by [18, Theorem 1.1]. We may therefore assume that $N$ is abelian and, in particular, $N$ is an elementary abelian $q$-group for some prime $q$.

We may also assume that $N$ is not semiregular that is, $N_{v} \neq 1$ for some vertex $v$. It follows from Lemma 2.1 that $1 \neq N_{v}^{\Gamma(v)} \unlhd G_{v}^{\Gamma(v)}$. As $\Gamma$ is $G$-arc-transitive, we have that $G_{v}^{\Gamma(v)}$ is transitive and so the orbits of $N_{v}^{\Gamma(v)}$ all have the same size, either 2 or $p$. Since $N$ is a $q$-group, this size is equal to $q$. Writing $d$ for the valency of $\Gamma / N$, we have that either $(d, q)=(2, p)$ or $(d, q)=(p, 2)$.

If $d=2$ and $q=p$, then it follows from [15, Theorem 1] that $\Gamma$ is isomorphic to the graph denoted by $\mathrm{C}(p, r, s)$ in [15]. By [15, Theorem 2.13], Aut $(\mathrm{C}(p, r, s))$ contains the nontrivial semiregular automorphism $\varrho$ defined in [15, Lemma 2.5].

We may thus assume that $d=p$ and $q=2$. In this case, if $u$ is adjacent to $v$, then $u$ has exactly $2=2 p / d$ neighbours in $v^{N}$. Let $K$ be the kernel of the action of $G$ on $N$-orbits. By the previous observation, the orbits of $K_{v}^{\Gamma(v)}$ have size 2 and thus it is a 2-group. It follows from Lemma 2.1 that $K_{v}$ is a 2-group and thus so is $K=N K_{v}$. 
Now, $G / K$ is an arc-transitive group of automorphisms of $\Gamma / N$, so we may apply Corollary 3.2. If $G / K$ has a semiregular element of odd prime order, then so does $G$, by Lemma 2.2. If $|\mathrm{V}(\Gamma / N)|$ is a power of 2, then so is $|\mathrm{V}(\Gamma)|$ and, in this case, $G$ contains a semiregular involution by Lemma 2.3. We may thus assume that we are in case (3) of Corollary 3.2 , that is, $G / K$ contains a normal 2 -subgroup $P / K$ such that $(\Gamma / P, G / P)$ is one of the graph-group pairs in (3-5) of Theorem 3.1. Note that $P$ is a 2-group. Let $M$ be a minimal normal subgroup of $G$ contained in the centre of $P$. Note that $M$ is an elementary abelian 2-group. We may assume that $M$ is not semiregular hence $M_{v} \neq 1$ and so by Lemma $2.1, M_{v}^{\Gamma(v)} \neq 1$. Moreover, $|M| \neq 2$ as otherwise $M_{v}=M$ and we would deduce that $M$ fixes each element of $\mathrm{V}(\Gamma)$, a contradiction. Since $M$ is central in $P, M_{v}$ fixes every vertex in $v^{P}$.

Note that the $G$-conjugates of $M_{v}$ must cover $M$, otherwise $M$ contains a nontrivial semiregular element. By the previous paragraph, the number of conjugates of $M_{v}$ is bounded above by the number of $P$-orbits, that is $|\mathrm{V}(\Gamma / P)|$, so we have

$$
|M| \leqslant\left|M_{v}\right||\mathrm{V}(\Gamma / P)| .
$$

Since $\Gamma$ is connected and $G$-arc-transitive, there are no edges within $P$-orbits. As $M_{v}^{\Gamma(v)} \neq 1$, there exists $g \in M_{v}$ such that $w$ and $w^{g}$ are distinct neighbours of $v$. Let $u$ be the other neighbour of $w$ in $v^{P}$. Since $M_{v}$ fixes every element of $v^{P}$ it follows that $u$ is also a neighbour of $w$ and $w^{g}$ and so $\left\{v, w, u, w^{g}\right\}$ is a 4-cycle in $\Gamma$. Thus the graph induced between adjacent $P$-orbits is a union of $\mathrm{C}_{4}$ 's.

If $x$ is a vertex and $y^{P}$ is a $P$-orbit adjacent to $x$, then there is a unique $\mathrm{C}_{4}$ containing $x$ between $x^{P}$ and $y^{P}$, and thus a unique vertex $z$ antipodal to $x$ in this $\mathrm{C}_{4}$. We say that $z$ is the buddy of $x$ with respect to $y^{P}$. The set of buddies of $v$ is equal to $\Gamma_{2}(v) \cap v^{P}$, which is clearly fixed setwise by $G_{v}$. Moreover, each vertex has the same number of buddies. Furthermore, since $G_{v}$ transitively permutes the set of $p P$-orbits adjacent to $v^{P}$, either $v$ has a unique buddy or it has exactly $p$ buddies.

If $v$ has a unique buddy $z$, then $\Gamma(v)=\Gamma(z)$, and so swapping every vertex with its unique buddy is a nontrivial semiregular automorphism. Thus it remains to consider the case where $v$ has $p$ buddies. We first prove the following.

Claim. If $X$ is a subgroup of $M$ that fixes pointwise both $a^{P}$ and $b^{P}$, and $c^{P}$ is a $P$-orbit adjacent to both $a^{P}$ and $b^{P}$, then $X$ fixes $c^{P}$ pointwise.

Proof. Suppose that some $x \in X$ does not fix $c$. Now $x$ fixes $a^{P}$ pointwise, so $c^{x}$ must be the buddy of $c$ with respect to $a^{P}$. Similarly, $c^{x}$ must be the buddy of $c$ with respect to $b^{P}$. These are distinct, which is a contradiction. It follows that $X$ fixes $c$ and, since $X \leqslant M$, also $c^{P}$.

Let $s \geqslant 1$, let $\alpha=\left(v_{0}, \ldots, v_{s}\right)$ be an $s$-arc of $\Gamma$ and let $\alpha^{\prime}=\left(v_{0}, \ldots, v_{s-1}\right)$. Now $\left|v_{s} M_{v_{s-1}}\right|=2$, so $\left|M_{v_{s-1}}: M_{v_{s-1} v_{s}}\right|=2$ and $\left|M_{\alpha^{\prime}}: M_{\alpha}\right| \leqslant 2$. Applying induction yields that

$$
\left|M_{v_{0}}: M_{\alpha}\right| \leqslant 2^{s}
$$

We first assume that $\Gamma / P$ and $G / P$ are as in (3) or (4) of Theorem 3.1. Let $\{u, v\}$ be an edge of $\Gamma$. By the previous paragraph, we have $\left|M_{v}: M_{u v}\right| \leqslant 2$. Recall that $M_{v}$ fixes all vertices in $v^{P}$, so $M_{u v}$ fixes all vertices in $v^{P} \cup u^{P}$. Combining the claim with Corollary 3.5 
yields that $M_{u v}=1$ and thus $\left|M_{v}\right|=2$. It follows that $|M| \leqslant\left|M_{v}\right||\mathrm{V}(\Gamma / P)|$ so $|M| \leqslant$ $2|\mathrm{~V}(\Gamma / P)|$. Since $M$ is minimal normal in $G$, it is an irreducible $G$-module over $\operatorname{GF}(2)$, of dimension at least two. In fact, since $M$ is central in $P$, it is also an irreducible $(G / P)$ module. Since $G / P$ is nonabelian simple or has a nonabelian simple group as an index two subgroup, this implies that $M$ is a faithful irreducible $(G / P)$-module over $\mathrm{GF}(2)$. If $G / P=\mathrm{M}_{11}$, then $|M| \geqslant 2^{10}$ [11, Theorem 8.1], contradicting $|M| \leqslant 2 \cdot 12=24$. If $G / P=\mathrm{PSL}_{2}(p)$ or $\mathrm{PGL}_{2}(p)$ then by [2, Section VIII], $|M| \geqslant 2^{(p-1) / 2}$. Recall that $p \geqslant 127$ and so this contradicts $|M| \leqslant 2\left(p^{2}-1\right) / 2 s<p^{2}-1$.

Finally, we assume that $\Gamma / P$ is in (5) of Theorem 3.1 , that is, $\Gamma / P$ is the canonical double cover of a graph $\Gamma^{\prime}$ which appears in (4) of Theorem 3.1. In particular, $\mathrm{V}(\Gamma / P)=$ $\mathrm{V}\left(\Gamma^{\prime}\right) \times\{0,1\}$. By Lemma 3.3, $\Gamma^{\prime}$ has a 3-cycle, say $(u, v, w)$. By Corollary 3.5 and Lemma 3.6, $\Gamma / P$ is dense with respect to $\{u, v\} \times\{0,1\}$. Now, let

$$
\bar{\alpha}=((u, 0),(v, 1),(w, 0),(u, 1),(v, 0)) \text {. }
$$

Since $\bar{\alpha}$ contains $\{u, v\} \times\{0,1\}, \Gamma / P$ is dense with respect to $\bar{\alpha}$. Note that $\bar{\alpha}$ is a 4 -arc of $\Gamma / P$. Let $\alpha$ be a 4 -arc of $\Gamma$ that projects to $\bar{\alpha}$. Since $\Gamma / P$ is dense with respect to $\bar{\alpha}$, arguing as in the last paragraph yields $M_{\alpha}=1$. On the other hand, if $v \in \mathrm{V}(\Gamma)$ is the the initial vertex of $\alpha$, then by (4.1), we have $\left|M_{v}: M_{\alpha}\right| \leqslant 2^{4}$ and thus $\left|M_{v}\right| \leqslant 2^{4}$. Since $|M| \leqslant$ $\left|M_{v}\right||\mathrm{V}(\Gamma / P)|$ it follows that $|M| \leqslant 2^{4}\left(p^{2}-1\right) / s$. As above, $M$ is a faithful irreducible $(G / P)$-module over GF $(2)$ of dimension at least two. Since $G / P=\mathrm{PGL}_{2}(p)$ we have from [2] that $|M| \geqslant 2^{(p-1) / 2}$, which again contradicts $|M| \leqslant 2^{4}\left(p^{2}-1\right) / s<2^{4}\left(p^{2}-1\right)$.

\section{ORCID iDs}

\section{Michael Giudici (D) https://orcid.org/0000-0001-5412-4656 \\ Gabriel Verret (D) https://orcid.org/0000-0003-1766-4834}

\section{References}

[1] M. Arezoomand, A. Abdollahi and P. Spiga, On problems concerning fixed-point-free permutations and on the polycirculant conjecture-a survey, Trans. Comb. 8 (2019), 15-40, doi: 10.22108/toc.2018.112665.1585.

[2] R. Burkhardt, Die Zerlegungsmatrizen der Gruppen PSL $\left(2, p^{f}\right)$, J. Algebra 40 (1976), 75-96, doi:10.1016/0021-8693(76)90088-0.

[3] T. C. Burness and M. Giudici, Permutation groups and derangements of odd prime order, $J$. Comb. Theory Ser. A 151 (2017), 102-130, doi:10.1016/j.jcta.2017.04.007.

[4] P. J. Cameron, M. Giudici, G. A. Jones, W. M. Kantor, M. H. Klin, D. Marušič and L. A. Nowitz, Transitive permutation groups without semiregular subgroups, J. London Math. Soc. (2) 66 (2002), 325-333, doi:10.1112/s0024610702003484.

[5] L. E. Dickson, Linear Groups: With an Exposition of the Galois Field Theory, Dover Publications, New York, 1958.

[6] J. D. Dixon and B. Mortimer, Permutation Groups, volume 163 of Graduate Texts in Mathematics, Springer-Verlag, New York, 1996, doi:10.1007/978-1-4612-0731-3.

[7] E. Dobson, A. Malnič, D. Marušič and L. A. Nowitz, Semiregular automorphisms of vertextransitive graphs of certain valencies, J. Comb. Theory Ser. B 97 (2007), 371-380, doi:10.1016/ j.jctb.2006.06.004. 
[8] M. Giudici, Quasiprimitive groups with no fixed point free elements of prime order, J. London Math. Soc. (2) 67 (2003), 73-84, doi:10.1112/s0024610702003812.

[9] M. Giudici, L. Morgan, P. Potočnik and G. Verret, Elusive groups of automorphisms of digraphs of small valency, European J. Combin. 46 (2015), 1-9, doi:10.1016/j.ejc.2014.11.004.

[10] M. Giudici and J. Xu, All vertex-transitive locally-quasiprimitive graphs have a semiregular automorphism, J. Algebraic Combin. 25 (2007), 217-232, doi:10.1007/s10801-006-0032-5.

[11] G. D. James, The modular characters of the Mathieu groups, J. Algebra 27 (1973), 57-111, doi:10.1016/0021-8693(73)90165-8.

[12] P. Lorimer, Vertex-transitive graphs: symmetric graphs of prime valency, J. Graph Theory 8 (1984), 55-68, doi:10.1002/jgt.3190080107.

[13] D. Marušič, On vertex symmetric digraphs, Discrete Math. 36 (1981), 69-81, doi:10.1016/ 0012-365x(81)90174-6.

[14] D. Marušič and R. Scapellato, Permutation groups, vertex-transitive digraphs and semiregular automorphisms, European J. Combin. 19 (1998), 707-712, doi:10.1006/eujc.1997.0192.

[15] C. E. Praeger and M. Y. Xu, A characterization of a class of symmetric graphs of twice prime valency, European J. Combin. 10 (1989), 91-102, doi:10.1016/s0195-6698(89)80037-x.

[16] G. Sabidussi, Vertex-transitive graphs, Monatsh. Math. 68 (1964), 426-438, doi:10.1007/ bf01304186.

[17] G. Verret, Arc-transitive graphs of valency 8 have a semiregular automorphism, Ars Math. Contemp. 8 (2015), 29-34, doi:10.26493/1855-3974.492.37d.

[18] J. Xu, Semiregular automorphisms of arc-transitive graphs with valency pq, European J. Combin. 29 (2008), 622-629, doi:10.1016/j.ejc.2007.04.008. 